\title{
THE FAR-ULTRAVIOLET SPECTRUM OF NGC 4151 AS OBSERVED WITH THE HOPKINS ULTRAVIOLET TELESCOPE ON ASTRO-2
}

\author{
Gerard A. Kriss, Arthur F. Davidsen, Wei Zheng, Jeffrey W. Kruk, and Brian R. Espey \\ Department of Physics and Astronomy, Johns Hopkins University, Baltimore, MD 21218 \\ Received 1995 July 3; accepted 1995 September 11
}

\begin{abstract}
We observed NGC 4151 on six separate occasions at intervals of 1 to 3 days using the Hopkins Ultraviolet Telescope during the flight of Astro-2 aboard the space shuttle Endeavour in 1995 March. The far-UV spectra cover the spectral range from the interstellar cutoff at 912 to $1840 \AA$ with a resolution of $2-4 \AA$. The mean spectrum, representing $4752 \mathrm{~s}$ of integration time, has a signal-to-noise ratio of approximately 30 , and it shows profound differences compared to that obtained during the flight of Astro- 1 in 1990 December. The continuum, $5.3 \times 10^{-13} \mathrm{erg} \mathrm{cm}^{-2} \mathrm{~s}^{-1} \AA^{-1}$ at $1455 \AA$, is 5 times brighter, the brightest UV flux ever observed for NGC 4151. All high-ionization absorption lines have strengthened considerably-S VI $\lambda \lambda 933,945, \mathrm{C}$ III $\lambda 977, \mathrm{O}$ VI $\lambda \lambda 1032$, 1038, N v $\lambda \lambda 1239,1243$, Si IV $\lambda \lambda 1394,1403$, and C IV $\lambda \lambda 1548,1551$. The Lyman series absorption lines have also increased in strength, with the bulk of the absorption requiring a neutral hydrogen column density of $5 \times 10^{17}$ $\mathrm{cm}^{-2}$ covering only $78 \%$ of the UV source with an effective Doppler parameter of $350 \mathrm{~km} \mathrm{~s}^{-1}$. However, up to $5 \times 10^{20} \mathrm{~cm}^{-2}$ of neutral hydrogen that fully covers the source could be present in gas with a thermal Doppler parameter of $20 \mathrm{~km} \mathrm{~s}^{-1}$. Single-zone photoionization models of warm absorbing gas are unable to account for both the X-ray absorbing material and the UV-absorbing gas, largely because of the wide range of ionization states present in the UV and the X-ray.

Subject headings: galaxies: active — galaxies: individual (NGC 4151) — galaxies: nuclei — galaxies: Seyfert — ultraviolet: galaxies
\end{abstract}

\section{INTRODUCTION}

The nearest and brightest active galactic nuclei (AGNs) naturally draw attention as the best places to study the structure of the central engine and its environs. While the bright Seyfert 1 galaxy NGC 4151 is atypical of most AGNs, it has a wide variety of unusual characteristics common to many-an extended narrow-line region (NLR) (Evans et al. 1993), rapid variability at optical, UV, and X-ray wavelengths (Antonucci \& Cohen 1983; Clavel et al. 1987; Perola et al. 1986), strong, broad absorption lines in the optical (Anderson 1974) and in the UV (Penston et al. 1981; Bromage et al. 1985; Kriss et al. 1992), and variable intrinsic X-ray absorption (Holt et al. 1980; Weaver et al. 1994a, b). Even though other AGNs exhibit one or more of these features, understanding them all in a self-consistent manner in NGC 4151 is a difficult task.

The biconical structure of the NLR in NGC 4151, a feature common to Seyfert 2 galaxies (Pogge 1989; Evans et al. 1994) and one that suggests that our line of sight to the ionizing continuum is obscured, led Evans et al. (1993) to propose that the UV-absorbing material, particularly the neutral hydrogen observed by Kriss et al. (1992), collimates the radiation. The total column density of the UV-absorbing gas (about $10^{19}-10^{21}$ $\mathrm{cm}^{-2}$, with a neutral column greater than $6 \times 10^{17} \mathrm{~cm}^{-2}$ ), however, is orders of magnitude below the reported X-ray columns of $10^{22}-10^{23} \mathrm{~cm}^{-2}$. While this X-ray absorption was originally characterized as a "leaky," presumably neutral, collection of gas clouds (Holt et al. 1980), the most recent high-resolution X-ray studies (Weaver et al. 1994a, b) suggest that the X-ray absorbing gas may be highly ionized, similar to the "warm" absorbers seen in other AGNs (e.g., Halpern 1984; Turner et al. 1993; Nandra \& Pounds 1994). If the gas is highly ionized, it may be possible to reconcile the disparate
UV and X-ray columns and find a common origin for the absorbing medium, as suggested for 3C 351 (Mathur et al. 1994).

Observations of NGC 4151 with the Hopkins Ultraviolet Telescope (HUT) during the flight of Astro-1 in 1990 revealed numerous features in the far-UV spectrum below $1200 \AA$, including $\mathrm{O}$ VI $\lambda \lambda 1032,1038$ and He II $\lambda 1085$ emission, and absorption by the hydrogen Lyman series as well as $\mathrm{O}$ VI $\lambda 1032,1038, \mathrm{C}$ III $\lambda 977$, and C III $\lambda 1176$. These lines provide valuable diagnostics for the density and ionization structure of the absorbing material. Given the rapid variability (days or less) historically seen in the UV in NGC 4151, we planned multiple pointings during the 16 day Astro- 2 mission to use the time variability as an additional tool for unraveling the physical characteristics of the line-emitting and line-absorbing clouds.

\section{OBSERVATIONS}

We observed NGC 4151 on six separate occasions with HUT during the course of the Astro-2 mission in 1995 March for a total of $4752 \mathrm{~s}$ in the combined spectrum. Each of the short integrations has a higher signal-to-noise ratio than the Astro-1 HUT observation of NGC 4151. Flux variations of about $10 \%$ as well as changes in absorption line strengths are seen among the six observations. We defer a detailed discussion of these short-timescale variations to a later paper. Here we present an analysis of the mean spectrum observed during the Astro- 2 mission and compare it to the Astro-1 observations of 1990 December.

The $0.9 \mathrm{~m}$ primary mirror of HUT collects light for a prime-focus, Rowland-circle spectrograph as described by Davidsen et al. (1992). First-order spectra recorded by the 


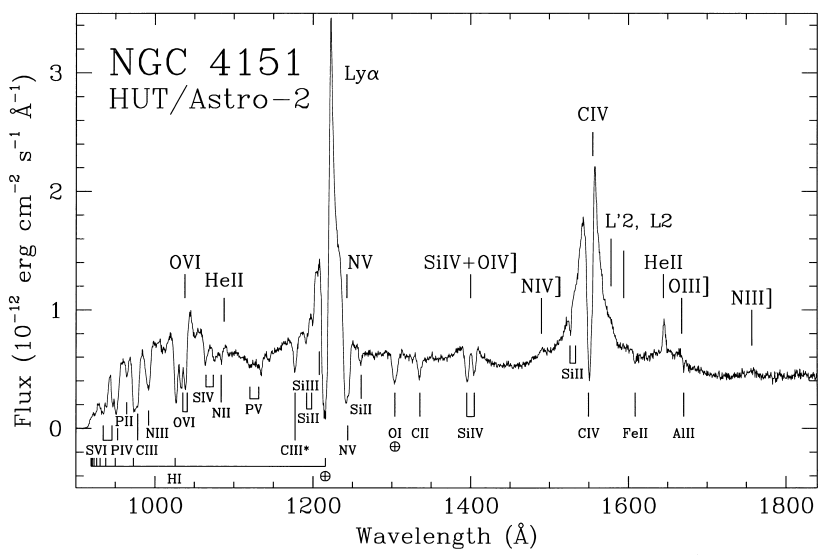

FIG. 1.-Flux-calibrated mean spectrum of NGC 4151 obtained with the HUT during the Astro-2 mission is shown. Prominent emission and absorption lines are marked. Earth symbol indicates features affected by geocoronal Ly $\alpha$ and $\mathrm{O}$ I emission.

photon-counting detector cover the 820-1840 Å spectral range with a sampling of about $0.52 \AA$ per pixel and a point-source resolution of about $3 \AA$. Absorption by neutral hydrogen in the interstellar medium effectively limits the shortest observable wavelengths in our spectra to more than $912 \AA$ Amprovements to HUT since the Astro-1 mission include silicon carbide coatings on the primary mirror and the spectrograph grating as well as a new detector system with a fresh CsI photocathode. These improvements and the performance of the telescope during the Astro-2 mission are described by Kruk et al. (1995).

Our data reduction uses IRAF 1 tasks developed for HUT. We determine the detector background and the uniform portion of the grating-scattered geocoronal $\mathrm{Ly} \alpha$ using regions free of airglow below the $912 \AA$ Lyman limit. The extended base of geocoronal $\operatorname{Ly} \alpha$ is removed by fitting the $\operatorname{Ly} \alpha$ region with a template profile derived from observations of blank fields during the mission. Weaker airglow lines are similarly removed with templates from blank fields. We then place the background-subtracted spectra on an absolute flux scale using the time-dependent inverse sensitivity curves developed from on-orbit observations and model atmospheres of white dwarfs as described by Kruk et al. (1995). A small correction for second-order light in the 1824-1840 $\AA$ region is made using the observed flux in the 912-920 A range. Statistical errors for each pixel are calculated from the raw count spectra assuming a Poisson distribution and are propagated through the data reduction process. The six separate observations (weighted by their exposure times) are combined to form the mean fluxcalibrated HUT spectrum of NGC 4151 shown in Figure 1.

We use the program SPECFIT in IRAF (Kriss 1994) to fit the continuum and to measure the emission and absorption lines. As in Kriss et al. (1992), we use a power law for the continuum, two components for the broad emission lines (a narrow Gaussian core and a broad base with a power-law profile), and single Gaussian profile absorption lines for all lines other than the Lyman series. We allow extinction to vary freely following a Cardelli, Clayton, \& Mathis (1989) curve with $R_{V}=3.1$.

Since the Lyman series absorption is anticipated to be

1 The Image Reduction and Analysis Facility (IRAF) is distributed by the National Optical Astronomy Observatories, which is operated by the Association of Universities for Research in Astronomy, Inc. (AURA), under cooperative agreement with the National Science Foundation. complex, with multiple blended components from the Galaxy and in NGC 4151, we compute grids of transmission functions including transitions up to $n=50$. Using Voigt profiles of varying column density, Doppler parameter, and redshift, we then convolve the transmission with the instrument resolution (a $3.0 \AA$ FWHM Gaussian) for use in the model fitting. Galactic neutral hydrogen is fixed at zero redshift at a column density of $2.0 \times 10^{20} \mathrm{~cm}^{-2}$ (Stark et al. 1992), but its Doppler parameter is allowed to vary freely. We allow two components for the hydrogen absorption in NGC 4151: gas with high Doppler parameters of several hundred kilometers per second as seen in the Astro-1 data (Kriss et al. 1992), and gas with low Doppler parameters of tens of kilometers per second characteristic of thermal gas. The redshift of these components is permitted to vary freely, as is the covering factor of the high Doppler parameter material. While the HUT spectrum has limited resolution, it contains much useful information that constrains the neutral hydrogen absorption properties: individual Lyman lines are visible through Lyman $\varepsilon$; the lines are broader than the instrument resolution; the Lyman limit is visible, and its position and sharpness are sensitive to the redshift, Doppler parameter, and column density of the absorbing gas.

Our best fit yields $\chi^{2} / \nu=2032 / 1516$ for 1683 data points between 912 and $1800 \AA$ (we omit a region $\pm 7.5 \AA$ in width surrounding geocoronal $\mathrm{Ly} \alpha$ ). While statistically unacceptable, the fit is strikingly good to the eye, and the high $\chi^{2}$ is largely a consequence of the high signal-to-noise ratio $(\mathrm{S} / \mathrm{N})$ and our inability to precisely model all instrumental features, such as remaining uncertainties in the flux calibration and the variation of resolution with wavelength.

The best-fit continuum has $f_{\lambda}=1.01 \times 10^{-12}(\lambda / 1000 \AA)^{-1.01}$ $\operatorname{erg~cm}{ }^{-2} \mathrm{~s}^{-1} \AA^{-1}$ with $E_{B-V}=0.04$. This extinction is consistent with the Astro-1 spectrum and with the Galactic extinction along that sight line (Burstein \& Heiles 1982). The continuum is bluer than that observed in 1990, when we found $f_{\lambda} \sim \lambda^{-0.50}$. Measured properties of absorption lines with equivalent widths greater than $1.0 \AA$ and secure identifications are given in Table 1. Our errors are dominated by systematic rather than statistical uncertainties, and so we do not include formal errors in the table. We estimate our measurements of equivalent widths and line widths to be accurate to about $10 \%$; velocity uncertainties are typically about $150 \mathrm{~km} \mathrm{~s}^{-1}$, and they are given relative to the systemic velocity of $997 \mathrm{~km} \mathrm{~s}^{-1}$ (Pedlar et al. 1992).

Most of the intrinsic Lyman line absorption in our fit is in a broad component that covers only $78 \% \pm 3 \%$ of the source with material at an effective Doppler parameter of $350 \pm 25$ $\mathrm{km} \mathrm{s}^{-1}$ and a relatively low neutral column density of $N_{\mathrm{H}}=4.8 \pm 0.5 \times 10^{17} \mathrm{~cm}^{-2}$. The low required column density results largely because of the short wavelength and relative sharpness of the Lyman limit. Higher columns, such as observed in the Astro-1 data, produce a cutoff at longer wavelengths, with a broad profile approaching the Lyman limit. Characteristics of the data that lead to such a high effective Doppler parameter are the intrinsic breadths of the Lyman lines, which are resolved, even at the HUT resolution, and the high relative strength of the higher order Lyman lines. The requirement that the gas only partially cover the source is also a consequence of the relative equivalent widths. The observed curve of growth for the Lyman lines is fairly flat, and partial covering permits column densities high enough to produce a 
TABLE 1

Absorption Lines in the Hopkins Ultraviolet Telescope SPECTRUM OF NGC 4151

\begin{tabular}{|c|c|c|c|c|}
\hline Line & $\begin{array}{l}\lambda_{\mathrm{vac}} \\
(\AA)\end{array}$ & $\begin{array}{l}\text { EW } \\
(\AA)\end{array}$ & $\begin{array}{l}\text { Velocity }^{\mathrm{a}} \\
\left(\mathrm{km} \mathrm{s}^{-1}\right)\end{array}$ & $\begin{array}{c}\text { FWHM } \\
\left(\mathrm{km} \mathrm{s}^{-1}\right)\end{array}$ \\
\hline S VI........... & 933.38 & 3.5 & -449 & 1419 \\
\hline Lye ............. & 937.80 & 2.9 & -646 & 1281 \\
\hline $\mathrm{S}$ vi............. & 944.52 & 3.0 & -446 & 1419 \\
\hline $\operatorname{Ly} \delta \ldots \ldots \ldots \ldots$ & 949.74 & 3.2 & -663 & 1368 \\
\hline $\operatorname{Ly} \gamma \ldots \ldots \ldots \ldots$ & 972.54 & 3.6 & -671 & 1420 \\
\hline $\mathrm{C}$ III $\ldots \ldots \ldots \ldots$ & 977.03 & 3.8 & -792 & 1516 \\
\hline $\mathrm{N}$ III $\ldots \ldots \ldots \ldots$ & 991.00 & 2.0 & -560 & 1293 \\
\hline $\operatorname{Ly} \beta \ldots \ldots \ldots \ldots$ & 1025.72 & 4.3 & -688 & 1492 \\
\hline $\mathrm{O}$ VI $\ldots \ldots \ldots \ldots$ & 1031.93 & 3.5 & -652 & 1437 \\
\hline $\mathrm{O}$ vi $\ldots \ldots \ldots \ldots$ & 1037.62 & 3.6 & -651 & 1437 \\
\hline $\mathrm{C}$ III ${ }^{*} \ldots \ldots \ldots$ & 1175.70 & 1.8 & -577 & 1193 \\
\hline Si II........... & 1193.14 & 1.1 & -1257 & 1264 \\
\hline $\mathrm{N}_{\mathrm{I}} \ldots \ldots \ldots \ldots \ldots$ & 1200.16 & 1.1 & -1138 & 1264 \\
\hline $\mathrm{N} v \ldots \ldots \ldots \ldots$ & 1240.15 & 8.8 & -420 & 2397 \\
\hline Si II ............ & 1260.42 & 1.1 & -1032 & 1264 \\
\hline $\mathrm{C}$ II $\ldots \ldots \ldots \ldots$ & 1335.30 & 1.4 & -987 & 1076 \\
\hline Si IV $\ldots \ldots \ldots \ldots$ & 1393.76 & 2.6 & -565 & 1181 \\
\hline Si IV ........... & 1402.77 & 2.1 & -572 & 1181 \\
\hline $\mathrm{C}$ IV $\ldots \ldots \ldots \ldots$ & 1548.19 & 5.7 & -546 & 1358 \\
\hline $\mathrm{C}$ IV $\ldots \ldots \ldots \ldots$ & 1550.77 & 3.1 & -547 & 1358 \\
\hline
\end{tabular}

a Velocity relative to a systemic redshift of $c z=997 \mathrm{~km} \mathrm{~s}^{-1}$.

flat curve of growth while simultaneously giving low enough equivalent widths to match the data.

The low column and partial covering of this broad component makes it transparent at the Lyman limit. The sharp cutoff in the spectrum must come from additional optically thick material with a fairly low Doppler parameter. The simplest model that matches the data is a purely Galactic column with $N_{\mathrm{H}}=2.0 \times 10^{20} \mathrm{~cm}^{-2}$ and $b=25 \mathrm{~km} \mathrm{~s}^{-1}$. However, this model is not unique since the position and sharpness of the Lyman limit are strongly coupled to the column density and the Doppler parameter. An equally good fit is provided by a Galactic column of $2.0 \times 10^{20} \mathrm{~cm}^{-2}$ at $b=10 \mathrm{~km} \mathrm{~s}^{-1}$ with an additional column of $2.0 \times 10^{20} \mathrm{~cm}^{-2}$ and $b=20 \mathrm{~km} \mathrm{~s}^{-1}$ at a redshift of $c z=15 \mathrm{~km} \mathrm{~s}^{-1}$. This excess column above Galactic could plausibly be associated with the outflowing absorbing material in NGC 4151. At $90 \%$ confidence this excess neutral column could be as high as $5.0 \times 10^{20} \mathrm{~cm}^{-2}$.

\section{DISCUSSION}

The observed flux of $5.3 \times 10^{-13} \mathrm{erg} \mathrm{cm}^{-2} \mathrm{~s}^{-1} \AA^{-1}$ at $1455 \AA$ is the brightest ever recorded for NGC 4151. The differences between the Astro- 1 and the Astro- 2 spectra are most clearly seen in the ratio of the Astro-1 spectrum to the mean Astro-2 spectrum shown in Figure 2. The continuum is 5 times brighter on average, obviously more blue than in 1990, and dramatically brighter approaching the Lyman limit. Ignoring the airglow features, the most prominent differences are the deeper absorption lines in the high ionization lines C IV $\lambda 1549$, $\mathrm{N} v \lambda 1240$, and $\mathrm{O}$ VI $\lambda 1034$ and in the hydrogen Lyman series. The narrow emission cores of He II $\lambda 1640$ and $\lambda 1085$ also stand out. While they are brighter now than in 1990, they have not brightened as much as the continuum or the cores of the broad emission lines, consistent with the suggestion of Clavel et al. (1987) that they form outside the BLR at a radius of about 250 lt-days. Perhaps the most interesting features are the broad, sharply peaked structures visible on the red wing of $\mathrm{C}$ IV $\lambda 1549$, Ly $\alpha$, and, to a lesser extent, $\mathrm{O}$ VI $\lambda 1034$. The peak redward of C IV matches the "satellite" lines L'2 and L2 first

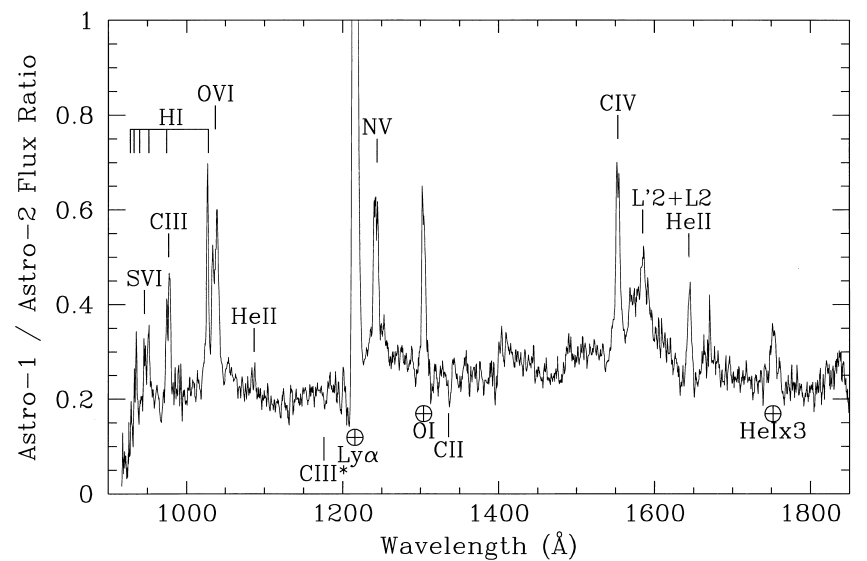

FIG. 2.-Ratio of the flux-calibrated HUT spectrum of NGC 4151 obtained during the Astro-1 mission to the mean HUT spectrum from Astro-2 is shown. The ratio has been smoothed with a boxcar filter of 3 pixels. Prominent features in the ratio are marked. Earth symbols indicate features affected by geocoronal Ly $\alpha, \mathrm{O}_{\mathrm{I}} \lambda 1304$, and third-order He I $\lambda 584$ emission.

reported by Ulrich et al. (1985), and the profile is quite similar to the C IV emission line itself.

Clavel et al. (1987) noted correlated variability between the C IV satellite lines although they do not correlate with the continuum. Given these characteristics and the observed redshifts and blueshifts, they suggested that the lines are formed in jets of material ejected from the nucleus. The L'2 and L2 features are present in both our Astro-1 and Astro- 2 spectra, but the L1 line is not. While they vary between our two observations, they are not in step with the rest of the broad line. The shape of the $\mathrm{L}^{\prime} 2+\mathrm{L} 2$ feature in the ratio of the Astro-1 to the Astro-2 data offers an intriguing possibility-the satellite line is not intrinsic jet emission, but rather a reflection of the broad-line region.

Features that are noticeably unchanged since Astro-1 are the absorption lines of the lowest ionization species, e.g., Si II $\lambda 1260$ and $C$ II $\lambda 1335$. Examining the velocities of the absorption lines in Table 1 shows two distinct groupings in velocity, one near $-600 \mathrm{~km} \mathrm{~s}^{-1}$ and another near $-1000 \mathrm{~km} \mathrm{~s}^{-1}$. The latter is comprised entirely of the lowest ionization species, and its velocity places it at rest relative to the Galaxy. The lack of variation (a point strongly argued by Veron, Veron-Cetty, \& Tarenghi 1985 using IUE data) and the zero redshift suggest that these lines are in our own interstellar medium rather than in NGC 4151.

Even though the Lyman lines show much stronger absorption in 1995 compared with 1990, the apparent column density of the gas responsible for the bulk of the absorption is now much lower. This implies that the hydrogen is more highly ionized and that turbulent velocities have increased, effectively increasing the Doppler parameter of the absorbers. The decrease in the neutral hydrogen column of the broad component of the Lyman lines compared to Astro-1 is consistent with photoionization of a warm absorber. An increase of a factor of 5 in the continuum intensity would lead to a 5 times decrease in the neutral column if the gas were optically thin in the Lyman continuum. Comparison with Astro-1 columns of greater than $6 \times 10^{17} \mathrm{~cm}^{-2}$ at a lower Doppler parameter of $200 \mathrm{~km} \mathrm{~s}^{-1}$ (Kriss et al. 1992) implies a decrease of at least $20 \%$ in the neutral column.

Given these conditions, is there a warm absorber model that can match the high X-ray columns and the observed UV 
ionization states? Ignoring the metal lines, one can certainly match the hydrogen column and the X-ray absorbing medium. Models of warm absorbers both in high-density clouds near the ionizing source (Netzer 1993) and in a more distant, lower density medium (Krolik \& Kriss 1995) can produce appropriately high total columns of $10^{23} \mathrm{~cm}^{-2}$ with low neutral columns. Photoionization models similar to those discussed by Krolik \& Kriss (1995) with $\Xi=10$, temperatures of a few times $10^{5} \mathrm{~K}$, and total columns of about $10^{23} \mathrm{~cm}^{-2}$ produce X-ray absorption typical of that historically seen in NGC 4151 (e.g., Weaver et al. 1994b) and match the absorption seen in C IV, $\mathrm{N} \mathrm{V}$, and $\mathrm{O}$ VI. However, whether the Doppler parameter is low and typical of thermal gas or turbulent and high as in the Lyman lines, it is hard to match simultaneously these high ionization lines with the relatively high neutral hydrogen column and the high columns observed for lower ionization ions like $\mathrm{C}$ III and Si IV.

Once again this implies an absorbing medium with multiple zones. While the complexity is inelegant, this is certainly not unexpected. Kriss et al. (1994b) argued that the UV absorption occurs in a thin atmosphere above the obscuring torus. One expects the medium in the vicinity of the photoionized skin of the torus to be turbulent and inhomogeneous (Balsara \& Krolik 1993). One could imagine clumps of high-density, lower temperature material suspended in a surrounding medium of higher temperature, lower density, more highly ionized gas. As material flows away from the torus and is accelerated into the X-ray heated wind, these higher density clumps will eventually evaporate into the surrounding hotter, lower density medium. Given the low opacity of the broad, turbulent component of the absorbing gas, it is unlikely that it collimates the radiation in NGC 4151. However, our data allow gas with thermal Doppler parameters of $10-20 \mathrm{~km} \mathrm{~s}^{-1}$ to be present in sufficient quantity to be opaque to the ionizing radiation.

This picture implies that our line of sight to NGC 4151 passes close to the surface of the obscuring torus itself, since the most profound difference between the warm absorber in NGC 4151 and others such as 3C 351 is the presence of the lower ionization C III lines, particularly the $\lambda 1176$ transition indicative of high-density gas. The UV-absorbing lines and $\mathrm{O}$ VIII edges seen in the X-ray in these other warm absorbers have lower total columns that may represent lower density gas more distant from the surface of the torus. Our future discussion of the shorter timescale variations seen in the UV-absorbing lines of NGC 4151 among the individual Astro-2 observations will give additional insights into the possible structure and location of the absorbing gas.

\section{SUMMARY}

Astro-2 observations of NGC 4151 with the HUT found this AGN at the highest UV flux ever observed, $5.3 \times 10^{-13}$ ergs $\mathrm{cm}^{-2} \mathrm{~s}^{-1} \AA^{-1}$ at $1455 \AA$. The spectrum shows significant differences compared to the 5 times less intense Astro-1 spectrum. The continuum is bluer, as is typical for AGNs in brighter states, and the opacity at the Lyman limit has decreased. Most of the Lyman line absorption requires a neutral hydrogen column of only $4.8 \pm 0.5 \times 10^{17} \mathrm{~cm}^{-2}$ at a Doppler parameter of $350 \pm 25 \mathrm{~km} \mathrm{~s}^{-1}$, and it appears to cover only $78 \% \pm 3 \%$ of the source. In spite of the lower intrinsic neutral column, the Lyman lines are significantly stronger in absorption as a consequence of the higher Doppler parameter. However, additional neutral hydrogen that fully covers the UV source with a column density of up to $5 \times 10^{20} \mathrm{~cm}^{-2}-$ sufficient to collimate the ionizing radiation-could also be present at thermal Doppler parameters of $20 \mathrm{~km} \mathrm{~s}^{-1}$. In addition to the stronger Lyman lines, all high ionization lines show much deeper absorption. These characteristics are all consistent with photoionization of the absorbing medium by the continuum source, but it is still difficult to find a common origin for both the UV and X-ray absorbing material. The broad range of observed ionization states cannot be matched with a single-zone photoionization model.

We are grateful to our colleagues at JHU and the Applied Physics Laboratory, who contributed so much to improving and preparing HUT for Astro-2. The expertise of the staffs at Kennedy Space Center and Marshall Space Flight Center contributed greatly to the success of the Astro-2 mission. J. Krolik and Z. Tsvetanov provided valuable comments on our new results. This work was supported by NASA contract NAS 5-27000 to the Johns Hopkins University.
Anderson, K. S. 1974, ApJ, 189, 195

Antonucci, R. R. J., \& Cohen, E. D. 1983, ApJ, 271, 564

Balsara, D. S., \& Krolik, J. H. 1993, ApJ, 402, 109

Bromage, G., et al. 1985, MNRAS, 215, 1

Burstein, D., \& Heiles, C. 1982, AJ, 87, 1165

Cardelli, J., Clayton, G., \& Mathis, J. 1989, ApJ, 345, 245

Clavel, J., et al. 1987, ApJ, 321, 251

Davidsen, A. F., et al. 1992, ApJ, 392, 264

Evans, I. N., Ford, H. C., Kriss, G. A., \& Tsvetanov, Z. 1994, in ASP Conf. Proc. 54, The First Stromlo Symposium: The Physics of Active Galaxies, ed. G. V. Bicknell, M. A. Dopita, \& P. J. Quinn (San Francisco: ASP), 3

Evans, I., Tsvetanov, Z., Kriss, G. A., Ford, H. C., Caganoff, S., \& Koratkar, A. P. 1993, ApJ, 417, 82

Halpern, J. 1984, ApJ, 281, 90

Holt, S. S., et al. 1980, ApJ, 241, L13

Kriss, G. A. 1994, in Astronomical Data Analysis Software and Systems III, ASP Conf. Proc. 61, ed. D. R. Crabtree, R. J. Hanisch, \& J. Barnes (San Francisco: ASP), 437

Kriss, G. A., et al. 1992, ApJ, 392, 485

Kriss, G. A., Tsvetanov, Z., \& Davidsen, A. F. 1994, in ASP Conf. Proc. 54, The First Stromlo Symposium: The Physics of Active Galaxies, ed. G. V. Bicknell, M. A. Dopita, \& P. J. Quinn (San Francisco: ASP), 281

\section{REFERENCES}

Krolik, J. H., \& Kriss, G. A. 1995, ApJ, 447, 512

Kruk, J. W., Durrance, S. T., Kriss, G. A., Davidsen, A. F., Blair, W. P., Espey, B. R., \& Finley, D. 1995, ApJ, 454, L1

Mathur, S., Wilkes, B., Elvis, M., \& Fiore, F. 1994, ApJ, 434, 493

Nandra, P., \& Pounds, K. A. 1994, MNRAS, 268, 405

Netzer, H. 1993, ApJ, 411, 594

Pedlar, A., Howley, P., Axon, D. J., \& Unger, S. W. 1992, MNRAS, 259, 369 Penston, M. V., et al. 1981, MNRAS, 196, 857

Perola, G. C., et al. 1986, ApJ, 306, 508

Pogge, R. 1989, ApJ, 345, 730

Stark, A. A., et al. 1992, ApJS, 79, 77

Turner, T. J., Nandra, K., George, I. M., Fabian, A. C., \& Pounds, K. A. 1993 ApJ, 419, 127

Ulrich, M. H., et al. 1985, Nature, 313, 745

Veron, P., Veron-Cetty, M. P., \& Tarenghi, M. 1985, A\&A, 150, 317

Weaver, K. A., et al. 1994a, ApJ, 423, 621

Weaver, K., Yaqoob, T., Holt, S. S., Mushotzky, R. F., Matsuoka, M., \& Yamauchi, M. 1994b, ApJ, 436, L27 